\title{
Estudo da Perda de Propagação através de ambientes de florestas usando um modelo híbrido com Equações Parabólicas
}

\author{
Fátima Nazaré Baraúna Magno, João Furtado de Souza, Zínia de Aquino Valente, Jessé Carvalho Costa, Gervásio \\ Protásio dos Santos Cavalcante. \\ Universidade Federal do Pará - Belém - Pará - Brasil
}

\begin{abstract}
Resumo - Neste trabalho é calculada a perda de propagação em um ambiente misto (floresta, ruas e edificações) utilizando-se o método de equações parabólicas com a adição de uma variável aleatória, o que torna o modelo híbrido. Para a resolução da equação parabólica é utilizado o esquema implícito de diferenças finitas de Crank-Nicolson. São considerados a propagação para $15^{\circ}$ na direção paraxial e para os parâmetros eletromagnéticos dos meios, o índice de refração complexo. Com o objetivo de validar o modelo proposto, foi feita uma campanha de medições em dois municípios do Estado do Pará, na cidade de Marituba na freqüência de $900 \mathrm{MHz}$ e na cidade de Barcarena em $1800 \mathrm{MHz}$. O modelo proposto foi então comparado aos dados experimentais. $O$ erro encontrado foi de $2,28 \mathrm{~dB}$ e o desvio padrão foi $3,38 \mathrm{~dB}$. Os resultados obtidos mostram a eficiência do método.
\end{abstract}

Palavras-Chave - Equação parabólica, Esquema de diferenças finitas, Floresta, Perda de propagação.

\begin{abstract}
This paper proposes a model to calculate the path loss propagation in a mixed environment (forest, streets and buildings) using the parabolic equations method and a random variable, which makes the hybrid model. To solve the parabolic equation the implicit finite differences CrankNicolson scheme was used. The propagation in the paraxial direction in $15^{\circ}$ and the complex index of refraction of the medium were considered. In order to validate the proposed model, a campaign of measurements was carried out in two districts of the State of Para, specifically in the city of Marituba in the frequency of $900 \mathrm{MHz}$ and in the city of Barcarena in $1800 \mathrm{MHz}$. The proposed model was then compared to the experimental data. The error found was 2.28 $\mathrm{dB}$ and standard deviation was $3.38 \mathrm{~dB}$. The results show the efficiency of the method.
\end{abstract}

Keywords - Finite difference scheme, Forest, Parabolic equation, Path loss.

Fátima N. B. Magno, João F. de Souza, Zínia de A. Valente, Departamento de Física; Jessé C. Costa, Departamento de Geofísica; Gervásio P. S. Cavalcante, Departamento de Engenharia Elétrica. Universidade Federal do Pará, Belém, Pará, Brasil. E-mails: fnbm@ufpa.br, furtado@ufpa.br, zinia@ufpa.br, jesse@ufpa.br, gervasio@ufpa.br.

\section{I.INTRODUÇÃO}

Nos sistemas de comunicações, particularmente para a comunicação sem fio, é de grande interesse o comportamento do rádio-enlace no que se refere a atenuação das ondas, desvanecimento e interferência co-canal. Quando há vegetação, tal como florestas, a comunicação é grandemente afetada devido aos múltiplos espalhamentos. A floresta causa grande atenuação nas ondas eletromagnéticas ao longo do percurso de rádio, reduzindo o alcance das comunicações. O efeito mais importante ocasionado pela floresta é o excesso de atenuação no sinal transmitido (ou perda de propagação), adicionado à perda no espaço livre. Sombreamento, espalhamento e absorção por árvores são algumas das causas da perda de propagação do sinal emitido em florestas. Por este motivo, a propagação dentro de vegetação deve ser bem estudada. Sabe-se que, quando o receptor está dentro da vegetação, as medidas da perda de propagação são caracterizadas por fortes flutuações, pois o desvanecimento é fortemente afetado devido às múltiplas flutuações. A perda de propagação é causada grandemente pelas folhas, troncos e galhos existentes no ambiente [1] [3].

Há três tipos de modelos para a predição do campo no interior de uma floresta: os empíricos, os semi-empíricos e os determinísticos. Uma das técnicas mais segura e amplamente utilizada é o método de equações parabólicas (EP). Inicialmente desenvolvido para o estudo de problemas acústicos, e mais tarde estendido para a propagação na troposfera. É comprovado também, poder ser este método usado para modelar o campo recebido em ambientes com bastante vegetação. O método de equações parabólicas é baseado na solução da equação de Helmholtz na direção paraxial [4]-[5].

O modelo apresentado neste trabalho é baseado no método de equações parabólicas para o cálculo da perda de propagação utilizando o esquema de diferenças finitas de Crank-Nicolson, considerando a propagação de ondas eletromagnéticas em ambiente misto (floresta cortada por ruas pavimentadas e edificações). Tem-se como objetivo calcular o campo recebido em dois ambientes do tipo mencionado, um no município de Barcarena e outro em 
Marituba, ambos localizados no Estado do Pará. O índice de refração utilizado neste trabalho é complexo e com valores diferenciados, dependendo da permissividade relativa e condutividade, levando-se em consideração os galhos e troncos das árvores, o material empregado na pavimentação das ruas, assim como o das várias edificações existentes nos locais. As freqüências utilizadas foram de $1,8 \mathrm{GHz}$ em Barcarena, e de $900 \mathrm{MHz}$ em Marituba; foi considerada a propagação em $15^{\circ}$ na direção paraxial para os dois ambientes. Com a finalidade de melhorar o modelo proposto foi adicionada a ele uma variável aleatória do tipo Rayleigh, o que o tornou híbrido (determinístico + estatístico), melhorando a sua precisão. Foi feita, então, a comparação entre a perda de propagação sem a variável aleatória, com esta variável e a perda obtida com os dados da campanha de medições e, finalmente, calculados os erros.

Este trabalho está organizado como segue: o modelo teórico, método de equações parabólicas (EP) e sua resolução pelo esquema de diferenças finitas de CrankNicolson, é descrito na secção II; na secção III é feita a descrição do ambiente utilizado; a perda de propagação calculada através do modelo híbrido é mostrada na seção IV; os resultados dos cálculos via equações parabólicas, modelo híbrido e dados experimentais, com suas respectivas figuras e tabelas representativas, são apresentados na secção V e na seção VI, a conclusão.

\section{O MÉTODO TEÓRICO}

A equação escalar de onda bidimensional pode ser escrita como [6]

$$
\frac{\partial^{2} \psi}{\partial x^{2}}+\frac{\partial^{2} \psi}{\partial z^{2}}+k^{2} n^{2} \psi=0
$$

onde $k$ é o número de onda e $n$ o índice de refração. Segundo Levy [7], escolhe-se $x$ na direção paraxial e faz-se

$$
\psi(x, z)=e^{i k x} u(x, z)
$$

obtendo-se a equação escalar

$$
\frac{\partial^{2} u}{\partial x^{2}}+\frac{\partial^{2} u}{\partial z^{2}}+2 i k \frac{\partial u}{\partial x}+k^{2}\left(n^{2}-1\right) u=0
$$

Esta equação pode ser escrita como

$$
\left(\frac{\partial}{\partial x}+i k(1-Q)\right)\left(\frac{\partial}{\partial x}+i k(1+Q)\right) u=0
$$

sendo

$$
\begin{aligned}
& Q=\sqrt{\frac{1}{k^{2}} \frac{\partial^{2}}{\partial z^{2}}+n^{2}(x, z)}=\sqrt{1+Z} \\
& Z=\frac{1}{k^{2}} \frac{\partial^{2}}{\partial z^{2}}+n^{2}(x, z)-1
\end{aligned}
$$

Nota-se que o operador $Z$ poderia representar uma quantidade pequena comparado à unidade.
A fatoração feita em (4) só é válida quando o operador $Q$ comuta com a derivada da distância $\partial / \partial x$, o que não acontece quando o índice de refração $n$ varia com a distância $x$. Entretanto, quando a variação é lenta, os erros resultantes desta fatoração são pequenos.

A equação (4) representa a propagação de ondas progressivas e regressivas, respectivamente, e a parte que representa a propagação de ondas progressivas é [7]

$$
\left(\frac{\partial}{\partial x}+i k(1-Q)\right) u=\left[\frac{\partial}{\partial x}+i k(1-\sqrt{1+Z})\right] u=0
$$

De (7) pode-se obter a equação parabólica padrão (EPP) utilizando-se a expansão de Taylor de primeira ordem de $\sqrt{1+Z}$, isto é $\sqrt{1+Z} \approx 1+Z / 2$. Através da equação parabólica padrão [8]

$$
\frac{\partial^{2} u}{\partial z^{2}}+2 i k \frac{\partial u}{\partial x}+k^{2}\left(n^{2}(x, z)-1\right) u=0
$$

Para encontrar-se (8) foi feita a aproximação válida para pequenos ângulos, no intervalo $15-25^{\circ}$ com a horizontal [9].

Discretizando-se (8) em $x$ e $z$ através do esquema de diferenças finitas de Crank-Nicolson [10], fazendo-se $\xi_{m}=\left(x_{m-1}+x_{m}\right) / 2$ o ponto médio na solução de $x_{\mathrm{m}-1}$ para $x_{\mathrm{m}}, \quad u_{j}^{m}=u\left(x_{m}, z_{j}\right), \quad b=4 i k\left(\Delta z^{2} / \Delta x\right) \quad \mathrm{e}$ $a_{j}^{m}=k^{2}\left(n^{2}\left(\xi_{m}, z_{j}\right)-1\right) \Delta z^{2}$, obtém-se [7]

$$
u_{j}^{m}\left(-2+b+a_{j}^{m}\right)+u_{j+1}^{m}+u_{j-1}^{m}=u_{j}^{m-1}\left(2+b-a_{j}^{m}\right)-u_{j+1}^{m-1}-u_{j-1}^{m-1}
$$

\section{O AMBIENTE DE TESTE}

O objetivo do modelo proposto, desenvolvido para estudar a propagação de ondas eletromagnéticas em ambientes mistos, é calcular o campo elétrico nos municípios de Marituba e Barcarena, no Estado do Pará.

Nestes dois locais encontram-se vegetações densas em algumas partes, cortadas por ruas pavimentadas e em outros locais há edificações.

As Figs. 1 e 2 mostram os ambientes de medições dos municípios de Barcarena e Marituba, respectivamente.

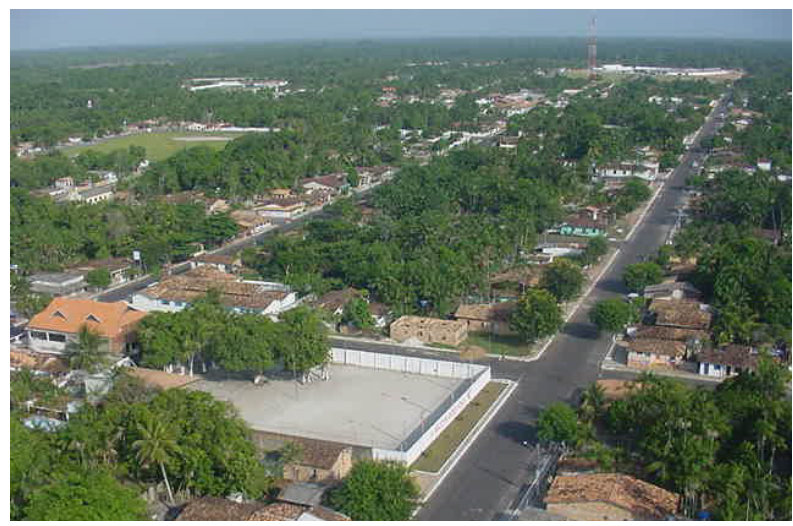

Fig. 1. Vista do município de Barcarena 


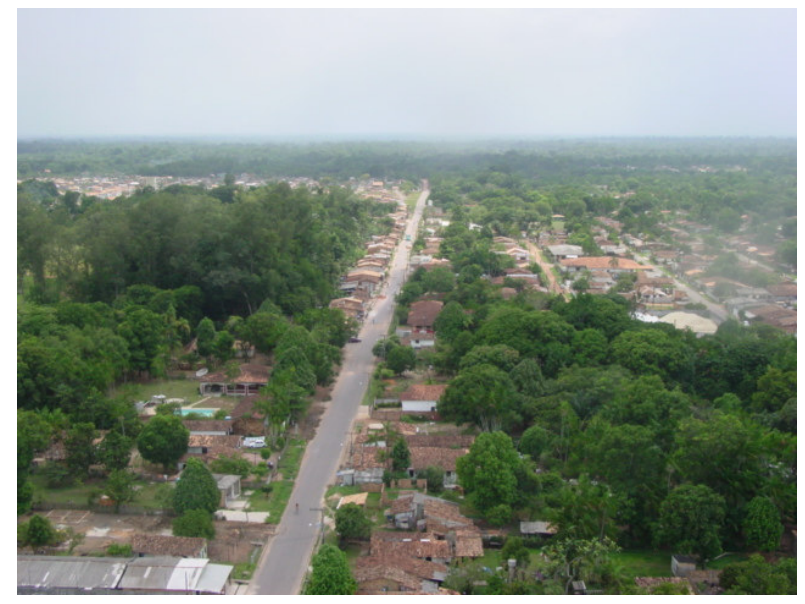

Fig. 2. Vista do município de Marituba.

A campanha de medições aconteceu em uma área de cobertura onde o sinal de rádio foi transmitido, separadamente, por estações fixas, uma localizada no município de Marituba e outra em Barcarena. Na campanha realizada em Marituba, a altura da antena transmissora foi fixada em $70 \mathrm{~m}, 44 \mathrm{~m}$ e $14 \mathrm{~m}$, sendo a última próxima à altura da copa das árvores. A antena utilizada foi uma omnidirecional com ganho de $2 \mathrm{dBi}$ irradiando um sinal $\mathrm{CW}$ em $900 \mathrm{MHz}$. Em Barcarena o sinal transmitido foi uma portadora em $1,8 \mathrm{GHz}$, com uma antena tipo painel a $60 \mathrm{~m}$ de altura, ganho de 17,5 dBi. Em ambos os municípios, o receptor móvel utilizou uma antena omnidirecional de ganho $3 \mathrm{dBi}$ fixada sobre um carro que percorreu algumas ruas da área de cobertura da Estação Rádio Base a uma velocidade de aproximadamente $30 \mathrm{~km} / \mathrm{h}$. Os resultados das medições foram registrados e processados posteriormente.

\section{PeRda de PropagaÇÃo calculada Via Modelo HÍBRIDO}

Para ser feita a simulação através do método de equações parabólicas (EP), considerou-se o sinal sendo transmitido por uma onda plana polarizada verticalmente; foram consideradas dimensões de comprimento e largura da vegetação e da rua, nos ambientes estudados. Foi escolhida a direção paraxial com aproximação para pequenos ângulos, até $15^{\circ}$.

Os parâmetros do meio apresentam características diferentes, sendo necessário que se utilizem os valores das várias constantes eletromagnéticas. O índice de refração foi considerado complexo, como dado por [11]. A Tabela I apresenta os valores da permissividade relativa e da condutividade empregadas na simulação do modelo proposto neste trabalho.

TABELA I

PERMISSIVIDADE RELATIVA E CONDUTIVIDADE

\begin{tabular}{|c||c|c|}
\hline Materiais & Permissividade Relativa & Condutividade $(\mathrm{mS} / \mathrm{m})$ \\
\hline \hline Floresta [12] & 1,10 & 0,1 \\
\hline Rua [12] & 2,70 & 90 \\
\hline Alvenaria das casas [13] & 5,18 & $32-40$ \\
\hline Madeira das casas [13] & 3,00 & 10 \\
\hline
\end{tabular}

\section{A. A Distribuição de Rayleigh}

Em um sistema de comunicação móvel, o sinal se propaga em um ambiente não estacionário. Os diferentes tipos de superfícies irregulares causam difração, dispersão, reflexão e absorção do sinal. $\mathrm{O}$ sinal transmitido interagindo com estas superfícies gera uma distribuição contínua deste sinal, onde amplitudes e fases variam de acordo com as propriedades eletromagnéticas destas superfícies. No receptor, o sinal chegará, então, através de múltiplos percursos.

Existe um grande número de Funções Distribuição de Probabilidade (FDP), que descrevem as características do sinal se propagando em um ambiente de comunicação móvel celular, constituindo assim modelos, onde as expressões encontradas representam as características do sinal durante o percurso. Neste trabalho foram testadas as mais utilizadas na literatura para comunicações móveis tais como, Rayleigh, Log-normal, Rice, Nakagami-m e Weibull. Através da análise estatística de erro a FDP de Rayleigh foi a que melhor se ajustou aos dados experimentais [14 - 15].

\section{B. A Perda de Propagação para o modelo Híbrido}

A perda de propagação foi calculada por [16] e será acrescida de uma variável aleatória para melhor ajustar o modelo às variações aleatórias do sinal

$$
L(d B)=36,57+20 \log _{10} f+20 \log _{10}\left|u_{0}\right|-20 \log _{10}|u|-G_{T}-G_{R}+X
$$

onde

$$
X=x \sigma
$$

sendo que, para (10), $u_{0}$ é o campo na distância de referência $\left(d_{0}\right), u$ o campo recebido, $f$ a freqüência expressa em $\mathrm{GHz}, G_{T}$ e $G_{R}$ os ganhos das antenas transmissora e receptora em $\mathrm{dB}$, respectivamente, e para (11), x são números aleatórios normalizados que caracterizam o comportamento da função distribuição encontrada em cada ambiente, e $\sigma$ o desvio-padrão dos dados medidos..

Foi implementado um programa em FORTRAN, para resolução de (9), e para os gráficos foi utilizado o Matlab 7.

\section{RESULTADOS NUMÉRICOS}

Na cidade de Marituba foram feitas medições usando antenas com três valores de alturas diferentes $(14 \mathrm{~m}, 44 \mathrm{~m}$, e $70 \mathrm{~m})$. Na cidade de Barcarena foram realizadas medições apenas em 60m. Para efeito de comparação do comportamento da perda de propagação, com relação a altura da antena transmissora, nos dois locais foi necessário fazer uma interpolação analítica nos dados de Marituba para simular a curva para uma antena de $60 \mathrm{~m}$

As curvas, em Marituba, para as três alturas de antenas do transmissor, e a obtida pela interpolação são mostradas na Fig. 3, onde se pode comprovar que a perda de propagação varia com a altura da antena transmissora. E se torna bastante grande para antenas muito baixas (neste caso a de $14 \mathrm{~m}$, mesma altura média da floresta). 


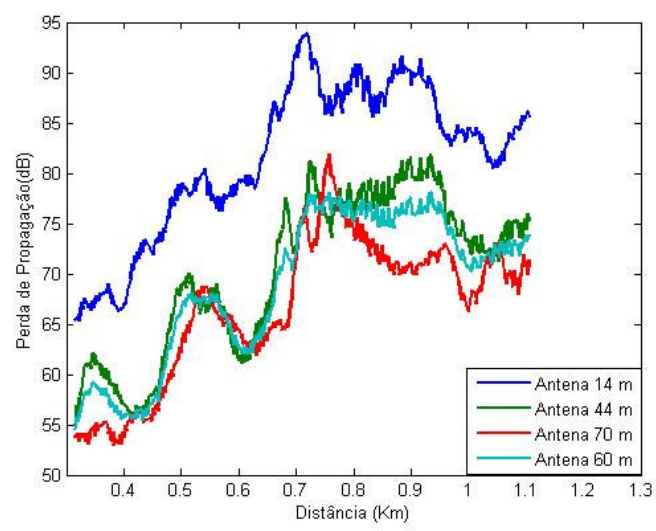

Fig. 3. Curvas para Marituba, para as alturas de antenas de 14, 44 e $70 \mathrm{~m}$, obtidas dos dados experimentais, e a de $60 \mathrm{~m}$, resultante da interpolação

A Fig. 4 mostra as curvas da perda de propagação, em decibéis, versus distância, em quilômetros, para o cálculo usando o método de equações parabólicas (EP) sem a variável aleatória e com esta variável, e valores medidos experimentalmente, no município de Barcarena.

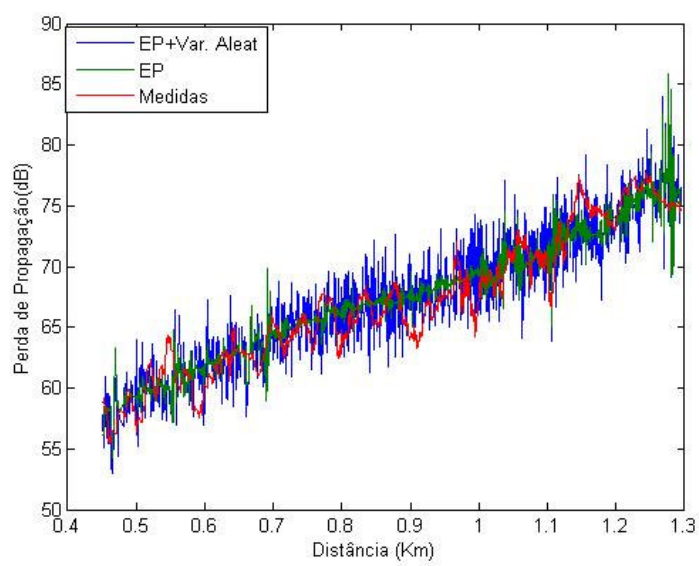

Fig. 4. Perda de propagação versus distância no município de Barcarena calculada via EP, EP+variável aleatória e experimentalmente.

Na Fig. 5 vê-se a perda de propagação versus distância, para o município de Marituba, calculada via equações parabólicas (EP), equações parabólicas + variável aleatória e dados simulados através de interpolação, para a antena com altura de $60 \mathrm{~m}$.

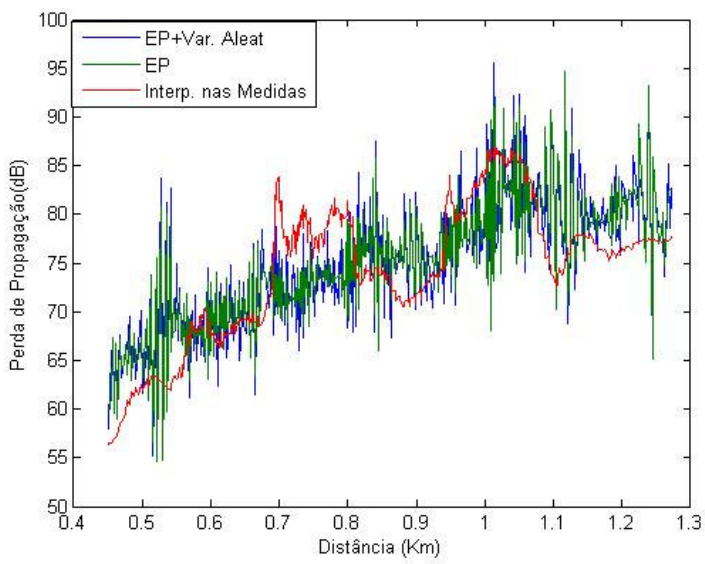

Fig. 5. Perda de propagação versus distância para o município de Marituba calculada via EP, EP+variável aleatória e dados simulados através de interpolação.
Analisando-se as curvas anteriores, nota-se a boa concordância do método proposto, de equações parabólicas com a adição da variável aleatória, aos dados experimentais.

A Tabela II fornece o erro médio, desvio padrão e erro rms para os municípios de Barcarena e Marituba, calculados para o modelo de equações parabólicas e para o modelo de equações parabólicas + variável aleatória, em relação às medidas experimentais e dados simulados através de interpolação. A diferença na estatística dos erros notada entre os municípios deve-se a não homogeneidade do local, a geometria do ambiente e a dificuldade em representar um modelo que se aproxime o máximo possível do ambiente real. É importante destacar que apesar da extrapolação utilizada para simular a altura de 60 metros para Marituba, os resultados foram coerentes. Observa-se ainda que a adição da variável aleatória permite caracterizar melhor a variabilidade do ambiente em estudo.

TABELA II

CÁLCULO DO ERRO MÉDIO, DESVIO PADRÃO E ERRO RMS

\begin{tabular}{|c||c|c|c|c|}
\hline Local & Modelo & $\begin{array}{c}\text { Erro } \\
(\mathrm{dB})\end{array}$ & $\begin{array}{c}\text { Desvio Padrão } \\
(\mathrm{dB})\end{array}$ & $\begin{array}{c}\text { Erro rms } \\
(\mathrm{dB})\end{array}$ \\
\hline \hline \multirow{2}{*}{ Barcarena } & EP & 2,04 & 2,03 & 3,06 \\
\cline { 2 - 5 } & $\begin{array}{c}\text { EP + Var. } \\
\text { Aleat. }\end{array}$ & 1,55 & 2,10 & 2,76 \\
\hline \multirow{2}{*}{ Marituba } & EP & 3,66 & 3,98 & 5,40 \\
\cline { 2 - 5 } & $\begin{array}{c}\text { EP + Var. } \\
\text { Aleat. }\end{array}$ & 3,01 & 4,66 & 5,56 \\
\hline
\end{tabular}

\section{CONCLUSÃO}

Neste trabalho foi utilizado um modelo determinístico, o método de equações parabólicas, adicionado a uma variável aleatória do tipo Rayleigh, para o estudo da perda de propagação em um ambiente misto, ou seja, floresta cortada por ruas pavimentadas e com edificações.

Utilizando-se o modelo de equações parabólicas resolvida pelo esquema implícito de diferenças finitas do tipo CrankNicolson nota-se grande rapidez no processamento dos dados. Para um microcomputador Pentium 4, 2,26 GHz, 1,48 GB de RAM, foi gasto aproximadamente 0,1 segundos. Isto se explica pelo fato de, no esquema de Crank-Nicolson, ter-se que resolver uma matriz tridiagonal. Isto é uma vantagem em relação a outros modelos existentes na literatura, onde se necessita de um grande tempo de computação. Este esquema também foi escolhido por ser incondicionalmente estável.

Para o cálculo do índice de refração foram consideradas as partes real e imaginária, o que aumenta a precisão do modelo proposto.

Os valores encontrados foram, em média, de 2,28 dB para o erro, 3,38 dB para o desvio padrão e, de 4,16 dB para o erro rms. Estes resultados mostram o bom comportamento do método de equações parabólicas com a adição da variável aleatória no estudo da perda de propagação em ambientes mistos, comparado às medidas experimentais.

Atualmente, estão sendo feitas simulações aumentando o ângulo de propagação para até $90^{\circ} \mathrm{com}$ a direção paraxial e, então, esta simulação será aplicada para este e outros ambientes. 
[1] M. Le Palud, "Propagation Modeling of VHF Radio Channel in Forest Environments", IEEE Military Communications Conference, pp. 609614,2004

[2] S. Phaiboon and S. Somkuarnpanit, "Mobile Path Loss Characteristics for Low Base Station Antenna Height in Different Forest Densities", IEEE, 2006.

[3] F. Wang and K. Sarabandi, "A Physics-Based Statistical Model for Wave Propagation Through Foliage", IEEE Transactions on Antennas and Propagation, Vol. 55, No. 3, March 2007.

[4] K. Arshad, F. Katsriku and A. Lasebae, "Radiowave VHF Propagation modelling in forest using finite elements", IEEE, pp. 2146-2149, 2006.

[5] G. M. Whitman, F. K. Schwering and M. Y. - C. Wu, "Collimated Beam Wave Pulse Propagation and Scattering in Vegetation Using Scalar Transport Theory", IEEE Transactions on Antennas and Propagation, Vol. 55, No. 3, June 2007.

[6] P. D. Holm, "Wide-Angle Shift-Map PE for a Piecewise Linear TerrainA Finite-Difference Approach", IEEE Transactions on Antennas and Propagation, Vol. 55, No. 10, October 2007.

[7] M. Levy, "Parabolic Equation Methods for Electromagnetic Wave Propagation", the Institution of Electrical Engineers, London, pp. 440, 2000.

[8]. K. H. Craig and M.F. Levy, "Parabolic Equation Modeling of the Effects of Multipath and Ducting on Radar Systems", IEE Proceedings-F, Vol. 138, No. 2, April 1991.

[9] A. Z. Hyaric, "Wide-Angle Nonlocal Boundary Conditions for the Parabolic Wave Equation", IEEE Transactions on Antennas and Propagation, Vol. 49, No. 6, June 2001.

[10] G. D. Smith, "Numerical Solution of Partial Differential Equations Finite Difference Methods", Oxford: Clarendon, 3rd edition, pp. 1138, 1984.

[11] R. K. Wangsness, "Electromagnetic Fields", John Wiley \& Sons, United States of America, pp. 34 - appendices, 1979.

[12] G. P. S. Cavalcante, M. A. R. Sanches, and R. A. N. Oliveira, "Mobile Radio Propagation along Mixed Paths in Forest Environment", Proceedings of IMOC'99, pp. 320-324, SBMO/IEEE, 1999.

[13] D. I. Axiotis and M. E. Theologou, "2 GHz Outdoor to Indoor Propagation at high Elevation Angles", IEEE, PIMRC 2002.

[14] J. D. Parsons, "Mobile Radio Propagation Channel" New York: Wiley, 2000

[15] M. D. Yacoub, "Foundations of Mobile Radio Engineering", CRC Press, pp. 92-95, 1993.

[16] L. W. Li, T. S. Yeo, P. S. Kooi, M. S. Leong, and J. H. Koh, "Analysis of Electromagnetic Wave Propagation in Forest Environment along Multiple Paths", Progress In Electromagnetics Research, PIER 23, pp. 137-164, 1999. 